\title{
Reaction Rate Maxima at Large Distances between Reactants
}

\author{
Martin Kuss-Petermann and Oliver S. Wenger*
}

\begin{abstract}
One commonly thinks that two reactants need to come very close to one another in order for a chemical reaction to occur. This is true for most reaction types, but electron transfer is an exception in this regard. It is a well-documented fact that electron transfers can occur over long distances $(\geq 15 \AA)$, but it is much less wellknown that theory predicts a regime in which electron transfer rates increase with increasing distance between reactants. This contribution explains the physical origin of this counter-intuitive behavior, and it identifies a set of conditions that might facilitate its experimental observation.
\end{abstract}

Keywords: Charge separation · Electronic coupling · Electron transfer · Marcus theory · Reorganization energy

\section{Introduction}

Chemical reactions commonly require collisional encounters between reactants. Electron transfer is an exception because the low mass of electrons permits longrange reactions between distant donors and acceptors. In many enzymes, electron transfer takes place between reactants that are separated by $\geq 15 \AA .{ }^{[1,2]}$ In artificial systems, electron transfer processes occurring over $\geq 25 \AA$ have been observed. ${ }^{[3]}$ With increasing donor-acceptor separation, electron transfer rates $\left(\mathrm{k}_{\mathrm{ET}}\right)$ usually decrease, ${ }^{[4]}$ but theory predicts a regime in which $\mathrm{k}_{\mathrm{ET}}$ increases with increasing distance. ${ }^{[5,6]}$ The purpose of this article is to introduce this counter-intuitive effect to a broader readership.

A well-known and experimentally very thoroughly investigated outcome of electron transfer theory is the so-called inverted driving-force effect (Fig. 1). ${ }^{[7]}$ At a given donor-acceptor distance $\left(\mathrm{r}_{\mathrm{DA}}\right)$, electron transfer rates increase with increasingly negative reaction free energy $\left(\Delta \mathrm{G}_{\mathrm{ET}}{ }^{0}\right)$ until they reach a maximum at which $-\Delta \mathrm{G}_{\mathrm{ET}}{ }^{0}$ is equal to the reorganization energy $(\lambda)$. Upon further increase of the driving-force, $\mathrm{k}_{\mathrm{ET}}$ decreases. This effect was initially pre-

\footnotetext{
${ }^{\star}$ Correspondence: Prof. Dr. O. S. Wenger Department of Chemistry

University of Basel

St. Johanns-Ring 19

$\mathrm{CH}-4056$ Basel

E-mail: oliver.wenger@unibas.ch
}

dicted by theory, ${ }^{[7,8]}$ and later verified by many experimental studies. ${ }^{[9-11]}$ The maximum in $\mathrm{k}_{\mathrm{ET}}$ at $-\Delta \mathrm{G}_{\mathrm{ET}}{ }^{0}=\lambda$ originates in the nuclear factor $\left(\kappa_{\mathrm{n}}\right)$ of the Marcus equation for electron transfer rates (Eqn. (1)). $\kappa_{\mathrm{n}}$ is evidently a Gaussian function of $\Delta \mathrm{G}_{\mathrm{ET}}{ }^{0}$, and it is called 'nuclear' factor because it captures the response of nuclei (of donor, acceptor, and solvent molecules) to the electron transfer event (in the form of the reorganization energy $\lambda$ ).

$$
\kappa_{n}=\exp \left(-\frac{\left(\lambda+\Delta G_{E T}{ }^{0}\right)^{2}}{4 \cdot \lambda \cdot k \cdot T}\right)
$$

$\kappa$ is equivalent to the activation en$\operatorname{ergy}\left(E_{A}\right)$ term of the Arrhenius equation with $\mathrm{E}_{\mathrm{A}}=\left(\lambda+\Delta \mathrm{G}_{\mathrm{ET}}{ }^{0}\right)^{2} /(4 \cdot \lambda)$. The reactant and product potential energy wells $\left(f_{r}, f_{p}\right)$ in Fig. 1 illustrate how $\mathrm{E}_{\mathrm{A}}$ goes through a minimum at $-\Delta \mathrm{G}_{\mathrm{ET}}{ }^{0}=\lambda$ where the reaction is in fact barrierless. In experiment, the decrease of $\mathrm{k}_{\mathrm{ET}}$ in the inverted regime $\left(-\Delta \mathrm{G}_{\mathrm{ET}}{ }^{0}>\lambda\right)$ is often less pronounced than expected based on Eqn. (1) due to so-called nuclear tunneling effects, ${ }^{[9]}$ but this is of minor importance here.

Aside from the nuclear factor $\left(\kappa_{n}\right)$, a frequency factor $\left(v_{n}\right)$, and an electronic factor $\left(\kappa_{\mathrm{el}}\right)$ govern $\mathrm{k}_{\mathrm{ET}}{ }^{\mathrm{n}}$ in the semi-classical limit (Eqn. (2)). ${ }^{[12]}$

$\mathrm{k}_{\mathrm{ET}}=\mathrm{v}_{\mathrm{n}} \cdot \kappa_{\mathrm{el}} \cdot \kappa_{\mathrm{n}}$

The frequency factor is equivalent to the collision frequency factor of the Arrhenius equation, whereas the electronic factor takes the interaction between distant donors and acceptors $\left(\mathrm{H}_{\mathrm{DA}}\right)$ into account.

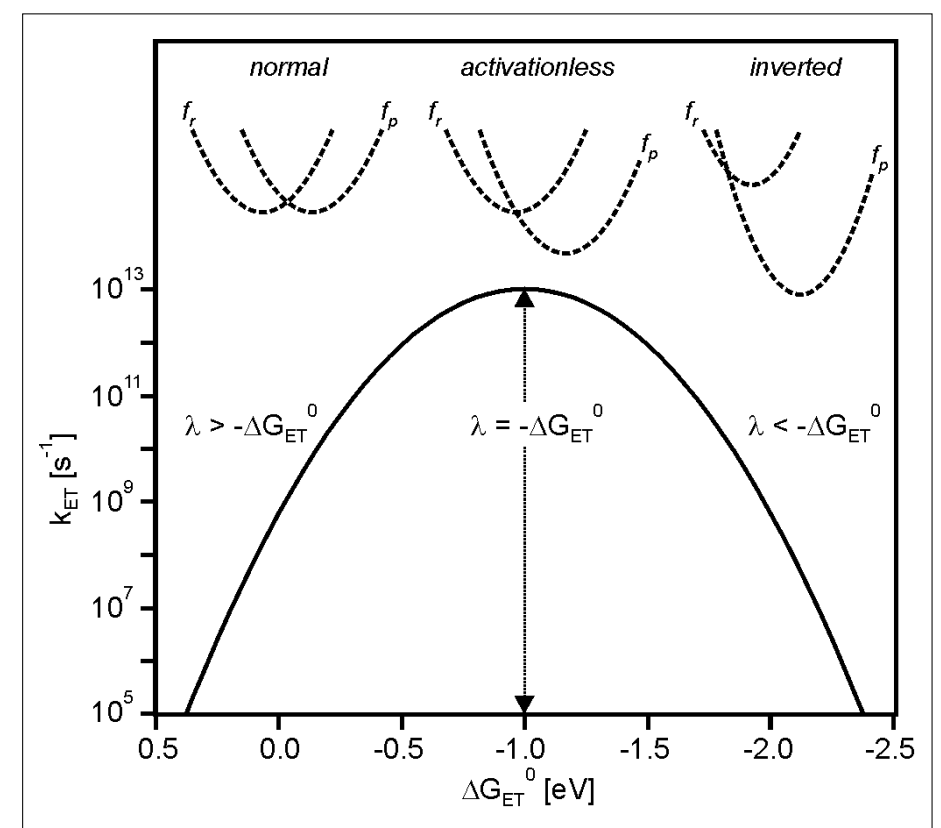

Fig. 1. Lower part: Dependence of electron transfer rates $\left(\mathrm{k}_{\mathrm{ET}}\right)$ on the reaction free energy $\left(\Delta G_{E T}{ }^{0}\right)$. The reorganization energy $(\lambda)$ was arbitrarily chosen as $1 \mathrm{eV}$ in this picture. Upper part: Reaction and product potential energy wells $\left(f_{r}, f_{p}\right)$ in the normal (left), activationless (middle), and the inverted regimes (right). The inverted driving-force effect is well explored and understood. 
$\mathrm{k}_{\mathrm{ET}}$ is in fact proportional to $\mathrm{H}_{\mathrm{DA}}{ }^{2}$ (Eqn. (3)).

$$
v_{n} \cdot \kappa_{e l}=\frac{2 \cdot H_{D A}{ }^{2}}{h} \cdot \sqrt{\frac{\pi^{3}}{\lambda \cdot k \cdot T}}
$$

According to superexchange theory, ${ }^{[13]}$ $\mathrm{H}_{\mathrm{DA}}$ can be nonzero even when donors and acceptors are separated by $\geq 15 \AA$ because bridging units (covalent linkers or intervening solvent molecules) can mediate long-range electronic coupling. Since hydrogen atom-like wavefunctions have radial parts that (at sufficiently large distance from the nuclei) drop off exponentially with increasing distance, orbital overlaps decrease exponentially with distance as well. This causes an exponential distance dependence of $\mathrm{H}_{\mathrm{DA}}$ (Eqn. (4)).

$$
H_{D A}\left(r_{D A}\right)=H_{D A}^{(0)} \cdot \exp \left(-\beta \cdot\left(r_{D A}-r_{0}\right)\right)
$$

In Eqn. (4), $\mathrm{H}_{\mathrm{DA}}{ }^{(0)}$ is the electronic coupling at contact distance $\left(\mathrm{r}_{0}\right)$ between reactants, and $\beta$ is the so-called distance decay parameter. Because $\mathrm{k}_{\mathrm{ET}} \propto \mathrm{H}_{\mathrm{DA}}{ }^{2}$ (Eqns (2), (3)), exponential distance dependences of $\mathrm{k}_{\mathrm{ET}}$ are frequently observed experimentally, both in biological and artificial systems. Exponential distance dependences are in fact typical for so-called superexchange tunneling, a process in which the electron tunnels through a barrier imposed by the intervening medium (covalent bridging units or solvent molecules) between the donor and the acceptor. ${ }^{[4]}$ When the intervening medium becomes redox-active itself, electrons (or holes) can hop over the barrier and $\mathrm{k}_{\mathrm{ET}}$ decreases less steeply with distance. ${ }^{[3,14]}$

Regardless of whether tunneling or hopping is considered, the common experimental observation is a decrease of $\mathrm{k}_{\mathrm{ET}}$ with increasing distance. One of the direct consequences of electron transfer theory is that there can be scenarios in which $\mathrm{k}_{\mathrm{ET}}$ increases with increasing distance, and this has been pointed out a long time ago. ${ }^{[5,6,15]}$ However, experimental evidence for this peculiar behavior is virtually non-existent, and consequently this effect is not well known. In the following chapters we will explain its physical origin, and we will identify a set of conditions that might favor its experimental observation.

\section{Distance Dependence of the Reorganization Energy $\lambda$}

The distance dependence of $\mathrm{k}_{\mathrm{ET}}$ is often solely attributed to the distance dependence of $\mathrm{H}_{\mathrm{DA}}$, and the fact that the reorganization energy $(\lambda)$ can change significantly with increasing donor-acceptor distance is frequently neglected. ${ }^{[16]}$ In most cases, this is unproblematic because the exponential distance dependence of $\mathrm{H}_{\mathrm{DA}}$ (Eqn. (4)) is dominant. However, there can be scenarios in which the distance dependence of the reorganization energy plays a decisive role. There are in fact two contributions to the overall reorganization energy, called inner- $\left(\lambda_{i}\right)$ and outer-sphere $\left(\lambda_{\mathrm{o}}\right)$ reorganization energies (Eqn. (5)). ${ }^{[7]}$

$$
\lambda=\lambda_{\mathrm{i}}+\lambda_{\mathrm{o}}
$$

The inner-sphere contribution describes the energy cost associated with nuclear reorganization occurring on the donor and acceptor moieties as a consequence of electron transfer, for example bond length changes. Solvent molecules around the donor and acceptor respond to the charge redistribution which is associated with electron transfer, and the energy which is necessary for solvent reorientation is the outer-sphere contribution to the overall reorganization energy. While $\lambda_{i}$ is essentially distance independent, $\lambda_{0}$ is a function of the donor-acceptor separation and solvent polarity. $\lambda$ frequently makes the dominant contribution to the overall reorganization energy.

In the simplest model describing the distance dependence of $\lambda$, electron donor and electron acceptor are assumed to be spheres with radii $a_{1}$ and $a_{2}$, separated by the distance $r_{D A}$ (Eqn. (6)).[7]

$$
\begin{aligned}
\lambda_{o}=(\Delta e)^{2} & \cdot\left(\frac{1}{2 \cdot a_{1}}+\frac{1}{2 \cdot a_{2}}-\frac{1}{r_{D A}}\right) \\
& \cdot\left(\frac{1}{D_{o p}}-\frac{1}{D_{s}}\right)
\end{aligned}
$$

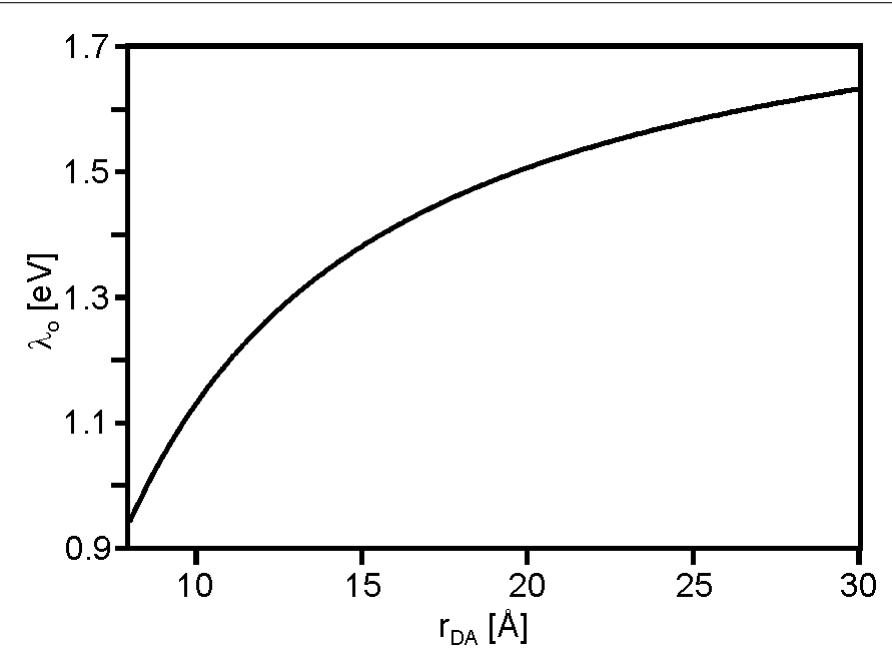

In Eqn. (6), $\mathrm{D}_{\mathrm{op}}$ and $\mathrm{D}_{\mathrm{s}}$ are the optical and static dielectric constants of the solvent, respectively. The former is connected to the refractive index $(\eta)$ by the relationship $D_{\text {op }}=\eta^{2}$. ( $D_{\text {op }}$ basically describes how solvent molecules respond to the oscillating electric field generated by optical wavelengths). From Eqn. (6) it is evident that $\lambda_{\mathrm{o}}$ increases with increasing $\mathrm{D}_{\mathrm{s}}$ (increasing solvent polarity) and with increasing $\mathrm{r}_{\mathrm{DA}}$. In Fig. 2, the distance dependence of $\lambda$ is shown for $\mathrm{CH}_{3} \mathrm{CN}\left(\eta=1.3341, \mathrm{D}_{\mathrm{s}}\right.$ $=35.7$ ) and reactant radii of $\mathrm{a}_{1}=\mathrm{a}_{2}=4 \AA$.

Under these conditions, Eqn. (6) predicts an increase of $\lambda_{\mathrm{o}}$ from $0.94 \mathrm{eV}$ to 1.63 eV between van-der-Waals contact $(8 \AA)$ and $30 \AA$. Qualitatively, the increase of $\lambda_{\text {o }}$ with increasing $r_{D A}$ can be understood on the basis of more isolated point charges resulting from electron transfer over longer distances, and consequently the solvent dipole - charge interaction increases with increasing donor-acceptor distance. There are also more sophisticated models that treat the reactants as ellipsoids, ${ }^{[17]}$ but the results in Fig. 2 are quite typical.

Thus, there are two opposing effects that influence the distance dependence of $\mathrm{k}_{\mathrm{ET}}$ : (i) the decrease of $\mathrm{H}_{\mathrm{DA}}$ with increasing distance (Eqn. (4)), and (ii) the increase of $\lambda_{\mathrm{o}}$ with increasing distance (Eqn. (6)).

\section{Consequences of Opposing Distance Dependences of $\mathrm{H}_{\mathrm{DA}}$ and $\lambda_{o}$ on Marcus Parabolas}

Since $\mathrm{k}_{\mathrm{ET}}$ is a function of $\Delta \mathrm{G}_{\mathrm{ET}}{ }^{0}, \lambda$, and $\mathrm{H}_{\mathrm{DA}}$, the influence of distant-dependent $\mathrm{H}_{\mathrm{DA}}$ and $\lambda_{\mathrm{o}}$ can be visualized in plots of $\log \left(\mathrm{k}_{\mathrm{ET}}\right)$ versus $\Delta \mathrm{G}_{\mathrm{ET}}{ }^{0}$, similar to the one shown in Fig. 1. Fig. 3 contains such plots for three different donor-acceptor distances $\left(\mathrm{r}_{\mathrm{DA}}=8,11,21 \AA\right)$, calculated using Eqns (1)-(6) and a few reasonable input parameters. In particular, a value of 200 $\mathrm{cm}^{-1}$ was assumed for $\mathrm{H}_{\mathrm{DA}}{ }^{(0)}$ and a value
Fig. 2. Outer-sphere reorganization energy $\left(\lambda_{0}\right)$ as a function donor-acceptor distance $\left(r_{\mathrm{DA}}\right)$ assuming spherical donors and acceptors with radii $\left(\mathrm{a}_{1}, \mathrm{a}_{2}\right)$ of $4 \AA$ and $\mathrm{CH}_{3} \mathrm{CN}(\eta=1.3341$, $\mathrm{D}_{\mathrm{s}}=35.7$ ) as the solvent. Calculated using Eqn. (6). 


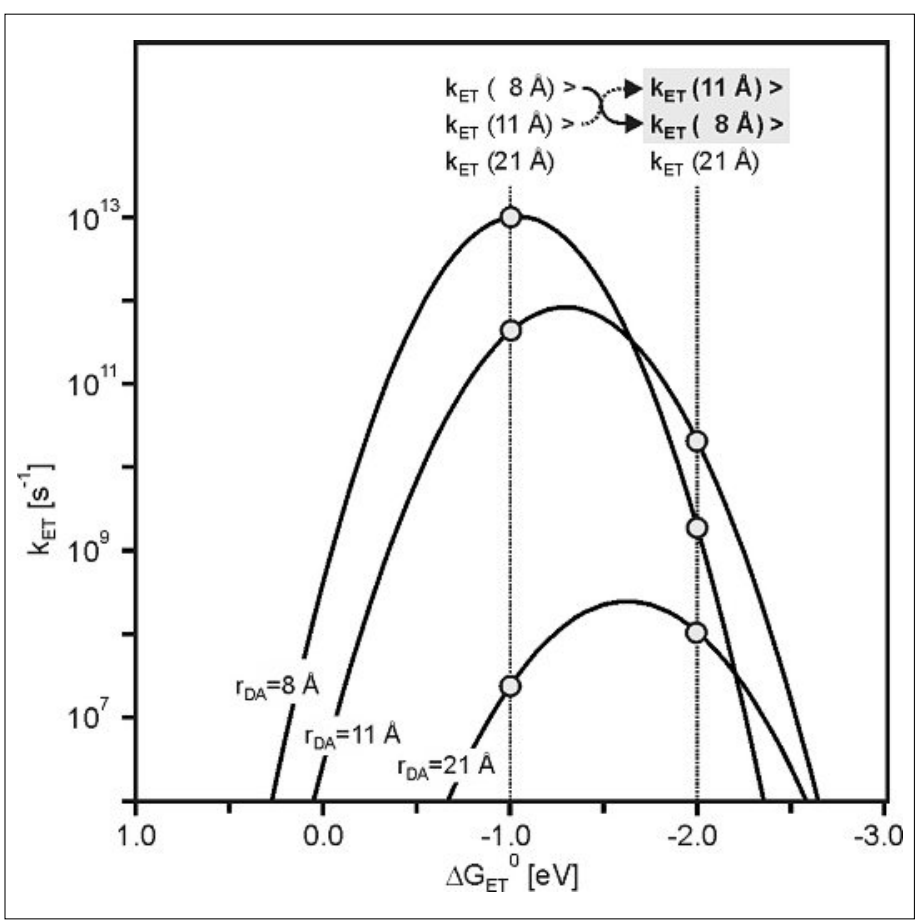

Fig. 3. Driving-force dependences of $k_{E T}$ for three different donor-acceptor distances $\left(r_{D A}\right)$. The calculations were performed using Eqns (1)-(6) and the following set of input parameters: $\mathrm{H}_{\mathrm{DA}}{ }^{(0)}=200$ $\mathrm{cm}^{-1}, \beta=0.8 \AA^{-1}, \lambda_{\mathrm{i}}=$ $0.1 \mathrm{eV}, \mathrm{a}_{1}=\mathrm{a}_{2}=4 \AA$, $\eta=1.3341\left(D_{o p}=\eta^{2}\right)$, $D_{s}=35.7$. The choice of these parameters is not unusual in any regard. of $0.8 \AA^{-1}$ was used for $\beta$. The calculations were made for reactants with radii of $4 \AA$ $\left(\mathrm{a}_{1}, \mathrm{a}_{2}\right)$ in $\mathrm{CH}_{3} \mathrm{CN}\left(\eta=1.3441, \mathrm{D}_{\mathrm{s}}=35.7\right)$. A constant inner-sphere reorganization energy $\left(\lambda_{\mathrm{i}}\right)$ of $0.1 \mathrm{eV}$ was assumed, a small value compared to the $\lambda_{\mathrm{o}}$ values calculated in Fig. 2.

The driving-force parabolas in Fig. 3 shift towards the bottom right corner as the donor-acceptor distance $\left(\mathrm{r}_{\mathrm{DA}}\right)$ gets longer, ${ }^{[18]}$ and it is easy to understand why: As $\mathrm{H}_{\mathrm{DA}}$ decreases with increasing $\mathrm{r}_{\mathrm{DA}}$ (Eqn. (4)), $\mathrm{k}_{\mathrm{ET}}$ must decrease because $\mathrm{k}_{\mathrm{ET}}$ $\propto \mathrm{H}_{\mathrm{DA}}{ }^{2}$ (Eqns (2), (3)), shifting the parabolas down. As $\lambda$ increases with increasing distance (Eqns (5), (6)), the maxima of the parabolas shift to the right because the barrierless point (i.e. maximal $\mathrm{k}_{\mathrm{ET}}$ ) is reached when $-\Delta \mathrm{G}_{\mathrm{ET}}{ }^{0}$ is equal to $\lambda$ (Eqn. (1), Fig. 1).

It is evident from Fig. 3 that at constant driving-force, very different distance dependences of $\mathrm{k}_{\mathrm{ET}}$ should be observable depending on the exact value of $\Delta \mathrm{G}_{\mathrm{ET}}{ }^{0}$. The two vertical dotted lines in Fig. 3 illustrate this aspect. The first line is drawn at $\Delta \mathrm{G}_{\mathrm{ET}}{ }^{0}$ $=-1.0 \mathrm{eV}$, which is close to the activationless point of the parabola calculated for $r_{\mathrm{DA}}=8 \AA$ but clearly in the normal regime $\left(-\Delta \mathrm{G}_{\mathrm{ET}}{ }^{0}<\lambda\right)$ for the two other parabolas $\left(\mathrm{r}_{\mathrm{DA}}=11,21 \AA\right)$. There is nothing peculiar to observe in this regime, $\mathrm{k}_{\mathrm{ET}}$ simply decreases with increasing distance. The second vertical line in Fig. 3 is drawn at $\Delta \mathrm{G}_{\mathrm{ET}}{ }^{0}=-2.0 \mathrm{eV}$, which is in the inverted region $\left(-\Delta \mathrm{G}_{\mathrm{ET}}{ }^{0}>\lambda\right)$ for all three parabolas. One immediately notices that $\mathrm{k}_{\mathrm{ET}}$ is now larger at $r_{D A}=11 \AA$ than at $r_{D A}=8 \AA$. In other words, the calculated rate for electron transfer increases with increasing donor-acceptor distance. It should be kept in mind that the y-axis in Fig. 3 is logarithmic hence the magnitude of this predicted effect is substantial.

\section{Distance Dependence of $k_{\mathrm{ET}}$ in Normal and Inverted Regimes}

As seen from Fig. 3, one expects fundamentally different distance dependences of $\mathrm{k}_{\mathrm{ET}}$ depending on whether a reaction occurs in the normal or inverted drivingforce regimes. It is useful to consider the two specific scenarios marked by the dotted vertical lines in Fig. 3 to get more quantitative insight.

The distance dependence of $\mathrm{k}_{\mathrm{ET}}$ at $\Delta \mathrm{G}_{\mathrm{ET}}{ }^{0}=-1.0 \mathrm{eV}$ is represented by the solid line in Fig. 4a. It was calculated using Eqns
(1)-(6) and the same input parameters as used above for Fig. $3\left(\mathrm{H}_{\mathrm{DA}}{ }^{(0)}=200 \mathrm{~cm}^{-1}\right.$, $\beta=0.8 \AA^{-1}, \lambda_{i}=0.1 \mathrm{eV}, \mathrm{a}_{1}=\mathrm{a}_{2}=4 \AA, \eta=$ $\left.1.3341, \mathrm{D}_{\mathrm{s}}=35.7\right)$. Under these conditions, $\mathrm{k}_{\mathrm{ET}}$ decreases nearly exponentially with increasing distance, with a minor deviation from strictly exponential decay behavior at short distances. This deviation stems from the distance dependence of the nuclear factor $\left(\kappa_{\mathrm{n}}\right)$ which is relatively weakly distant dependent at $\Delta \mathrm{G}_{\mathrm{ET}}{ }^{0}=-1.0 \mathrm{eV}$ (dashed line in Fig. 4a). The dominant contribution to $\mathrm{k}_{\mathrm{ET}}$ comes from the $v_{\mathrm{n}} \cdot \kappa_{\mathrm{el}}$ term (dotted line in Fig. 4a) at all distances. In other words, the distance dependence of $\mathrm{H}_{\mathrm{DA}}$ (Eqn. (6)) dominates over the distance dependence of $\lambda_{\mathrm{o}}$ (Eqn. (4)) at $\Delta \mathrm{G}_{\mathrm{ET}}{ }^{0}=-1.0 \mathrm{eV}$, and consequently one observes the commonly expected nearly exponential distance dependence of $\mathrm{k}_{\mathrm{ET}}$ in this regime.

In the inverted driving-force regime at $\Delta \mathrm{G}_{\mathrm{ET}}{ }^{0}=-2.0 \mathrm{eV}$, the distance dependence of the nuclear factor $\left(\kappa_{\mathrm{n}}\right)$ begins to play a decisive role, particularly at short distances (dashed line in Fig. $4 b$ ). Now $\kappa_{n}$ exhibits qualitatively different behavior from the situation at $\Delta \mathrm{G}_{\mathrm{ET}}{ }^{0}=-1.0 \mathrm{eV}$ in that $\kappa_{\mathrm{n}}$ increases with increasing distance. The reason for this is that the $\left(\lambda+\Delta \mathrm{G}_{\mathrm{ET}}{ }^{0}\right)^{2}$ term in $\kappa_{\mathrm{n}}$ (Eqn. (1)) decreases with increasing $\lambda$ when $-\Delta \mathrm{G}_{\mathrm{ET}}{ }^{0}>\lambda$ (the sum of $\lambda$ and $\Delta \mathrm{G}_{\mathrm{ET}}{ }^{0}$ becomes less negative). The increase of $\kappa_{n}$ is particularly steep at relatively short distances, because in this regime the distance dependence of $\lambda_{0}$ is steepest (Fig. 2). Over the distance range between 8 and $11 \AA, \kappa_{n}$ increases by more than two orders of magnitude (dashed line in Fig. 4b), while the decrease of $v_{n} \cdot \kappa_{e l}$ over the same distance range (dotted line in Fig. 4b) is close to one order of magnitude. Since $k_{\mathrm{ET}}$ is the product of $\kappa_{\mathrm{n}}$ and $\boldsymbol{v}_{\mathrm{n}} \cdot \kappa_{\mathrm{el}}$ (Eqn. (2)), the consequence is a maximum of $\mathrm{k}_{\mathrm{ET}}$ at $11 \AA$ (solid line in Fig. 4b), significantly beyond van-der-Waals contact distance ( 8

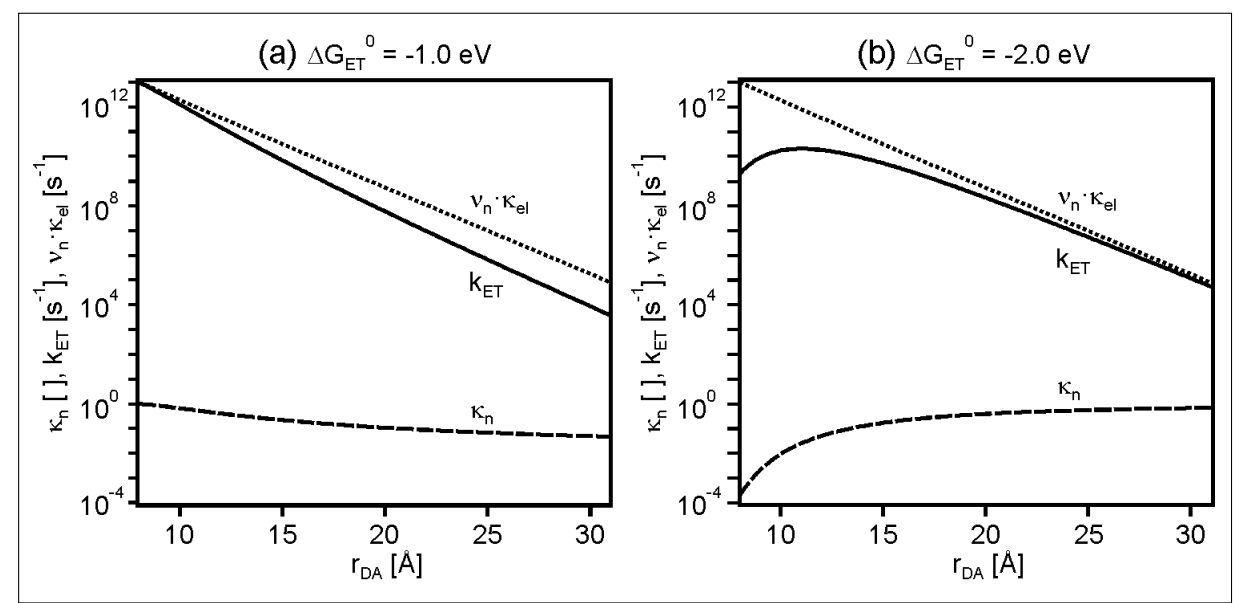

Fig. 4. Distance dependences of $k_{E T}$ (solid lines), the nuclear factor $\kappa_{n}$ (dashed lines), and the product of frequency factor and electronic factor $v_{n} \cdot \kappa_{e l}$ (dotted lines). The calculations are based on Eqns (1)-(6) and the same set of input parameters as for Fig. 3. The curves in (a) were calculated for $\Delta \mathrm{G}_{\mathrm{ET}}{ }^{0}=-1.0 \mathrm{eV}$, the curves in (b) were obtained for $\Delta \mathrm{G}_{\mathrm{ET}}{ }^{0}=-2.0 \mathrm{eV}$ (i.e. the two drivingforces marked by dotted vertical lines in Fig. 3). 
$\AA$ ). Thus, between 8 and $11 \AA$, the distance dependence of $\mathrm{k}_{\mathrm{ET}}$ is dominated by the distance dependence of $\lambda$, and the electronic coupling $\left(\mathrm{H}_{\mathrm{DA}}\right)$ only plays a dominant role at longer distances.

The solid lines from Fig. 4 are shown on linear scales in Fig. 5. The difference between the distance dependences of $\mathrm{k}_{\mathrm{ET}}$ at the two different driving-forces $\left(\Delta \mathrm{G}_{\mathrm{ET}} \stackrel{0 \mathrm{ET}}{=}\right.$ $-1.0 \mathrm{eV}$ dotted line; $\Delta \mathrm{G}_{\mathrm{ET}}{ }^{0}=-2.0 \mathrm{eV}$ solid line) becomes particularly evident in this representation. Obviously, the precise location of the rate maxima is dependent on the specific properties of the donor/solvent/ acceptor combination, but, as noted above, the set of input parameters that leads to the calculation of the curves in Fig. 5 is by no means unusual.

The increase of the nuclear factor with increasing distance at $\Delta \mathrm{G}_{\mathrm{ET}}{ }^{0}=-2.0 \mathrm{eV}$ can easily be rationalized on the basis of the Marcus parabola in Fig. 3. The corresponding vertical line intersects the parabola for $\mathrm{r}_{\mathrm{DA}}=8 \AA$ deeply in the inverted region where $-\Delta \mathrm{G}_{\mathrm{ET}}{ }^{0}$ is significantly greater than $\lambda$. As $r_{D A}$ increases, the intersection occurs at less and less deeply inverted points because the parabola shift to the right. This leads to reactant $\left(f_{r}\right)$ and product $\left(f_{p}\right)$ potential energy wells as illustrated in Fig. 6. At $r_{D A}=8 \AA$ (Fig. 6a), one is clearly in the inverted region in which the product potential well intersects the reactant potential well to the left of the minimum of the latter. Already at $r_{D A}=11 \AA$ (Fig. 6b), the intersection between the two potential wells occurs significantly closer to the minimum of the reactant well, and thus the activation barrier for electron transfer decreases. This accounts for the rate increase between 8 and $11 \AA$. At $r_{D A}=21 \AA$, the activation barrier gets even lower (Fig. 6c), but at that point the decrease in $\mathrm{H}_{\mathrm{DA}}$ is already dominant.

\section{Practical Implications of the Unusual Distance Dependence of $\mathbf{k}_{\mathrm{ET}}$}

The counter-intuitive distance dependence of reaction rates illustrated by Fig. 5 is certainly of fundamental interest, but of course the question arises what practical implications this effect might have. The answer is simple: Many photoinduced electron transfer reactions that lead to the generation of electron-hole pairs, a form of chemically stored light energy, occur in the normal regime in which $-\Delta \mathrm{G}_{\mathrm{ET}}{ }^{0}<\lambda$. Once these electron-hole pairs are formed, thermal reverse electron transfer processes leading to a recombination of electron and hole can occur, and such reactions are obviously undesired if light is to be converted into chemically useful forms of energy. Such charge-recombination events

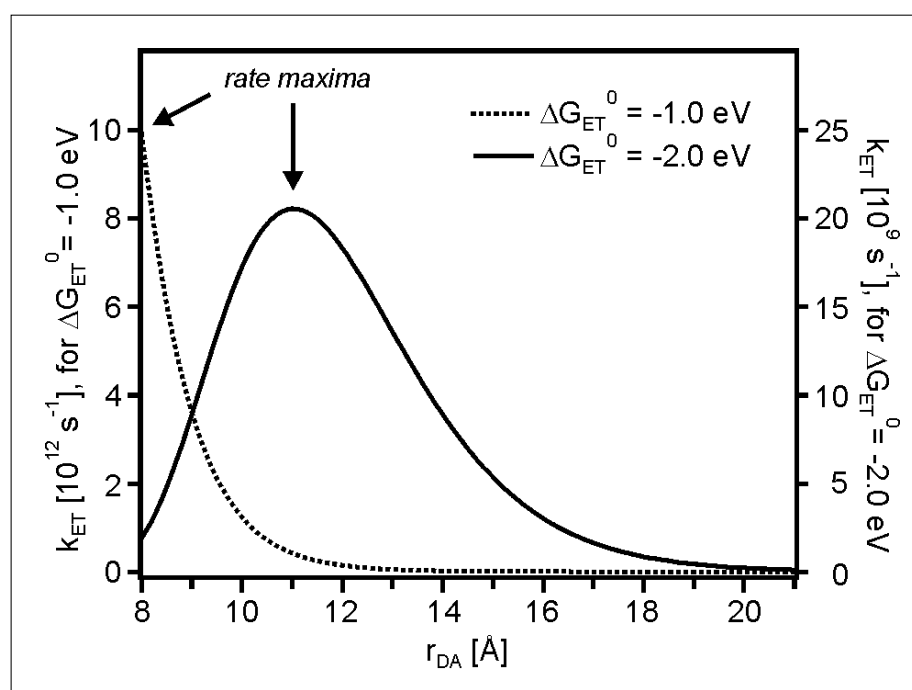

Fig. 5. Distance dependence of $\mathrm{k}_{\mathrm{ET}}$ in the normal (dotted line; $\Delta \mathrm{G}_{\mathrm{ET}}{ }^{0}=-1.0 \mathrm{eV}$ ) and the inverted (solid line; $\Delta \mathrm{G}_{\mathrm{ET}}{ }^{0}=-2.0 \mathrm{eV}$ ) driving-force regimes. The calculations are based on Eqns (1)-(6) and the same set of input parameters as for Figs 3 and 4.

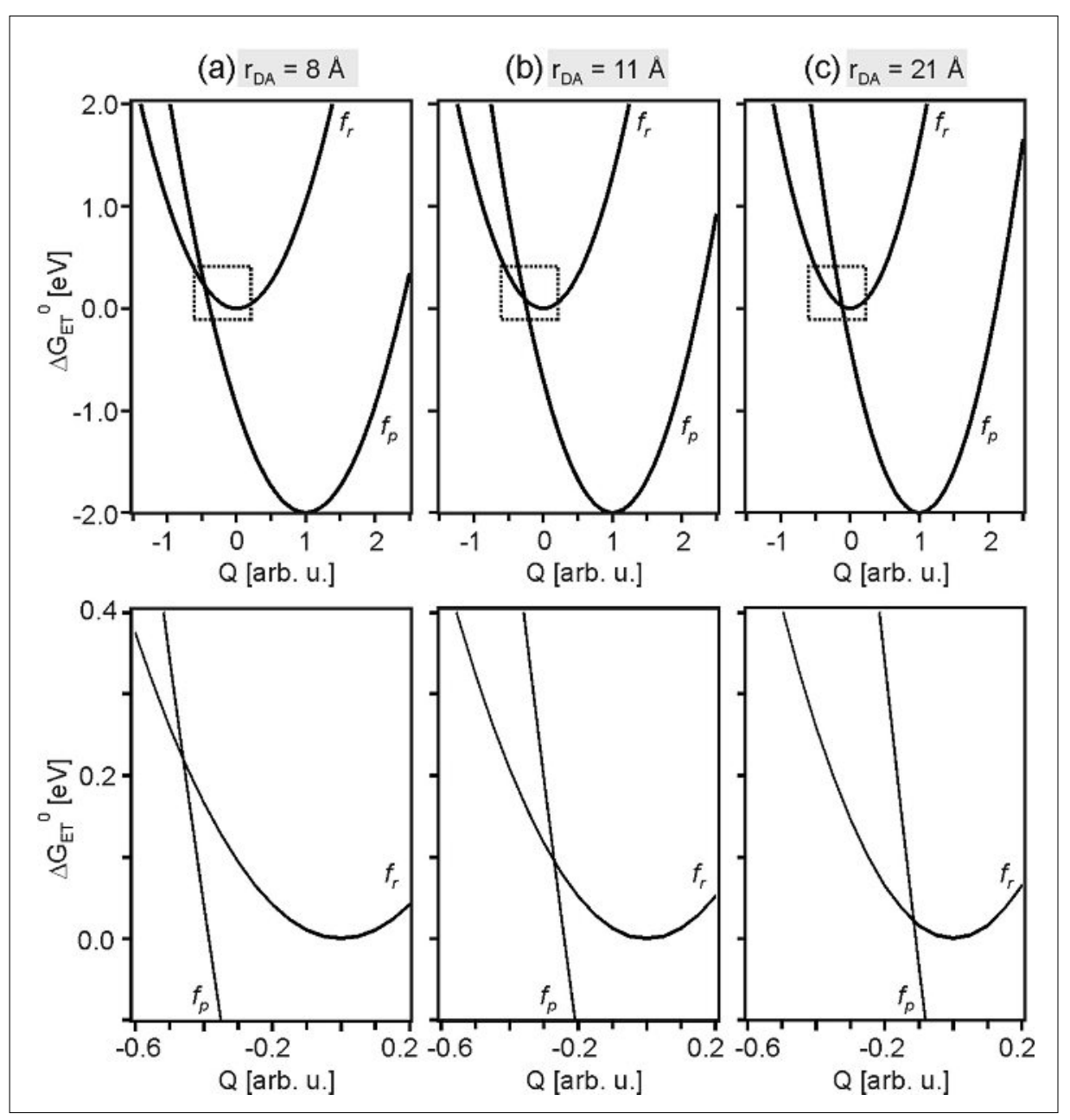

Fig. 6. Upper half: Reactant $\left(\mathrm{f}_{\mathrm{r}}\right)$ and product $\left(\mathrm{f}_{\mathrm{p}}\right)$ potential energy wells calculated on the basis of the parameters used in Figs 3 and $4 \mathrm{~b}\left(\Delta \mathrm{G}_{\mathrm{ET}}{ }^{0}=-2.0 \mathrm{eV}\right) . \mathrm{Q}$ is a normal coordinate in arbitrary units, capturing all nuclear changes (on donor, acceptor, and solvent molecules). Lower half: Zooms into the regions marked by the dotted squares in the upper half.

frequently occur in the inverted regime in which $-\Delta \mathrm{G}_{\mathrm{ET}}{ }^{0}>\lambda$. Fig. 5 illustrates how this set of conditions can be problematic for obtaining efficient photoinduced charge separation in bimolecular reaction systems: The desired photoinduced process has its rate maximum at close contact between reactants (dotted line), because this process occurs in the normal regime. Once the products have been formed, they can diffuse away from each other, but as the distance between them increases, the rate for the undesired charge-recombination event actually increases because this process occurs in the inverted regime (solid line in Fig. 5). Consequently, the effect 
illustrated by Fig. 5 can severely limit the quantum efficiency of light-to-chemical energy conversion. The effect outlined above has been used as an argument to explain the difficulties in observing the inverted driving-force effect in bimolecular reaction systems. ${ }^{[6,15]}$

\section{Experimental Studies of Unusual Distance Dependences of $k_{E T}$}

We are unaware of prior experimental studies which have provided direct evidence for an electron transfer rate maximum at large donor-acceptor separations due to the effect described above. As noted in the introduction, the phenomenon itself has been predicted a long time ago. $[5,6,15]$ Also, the increase of $\lambda_{0}$ with increasing distance is well documented, and of course the exponential decrease of $\mathrm{H}_{\mathrm{DA}}$ has been observed numerous times. ${ }^{[4]}$ However, it is not trivial to find unambiguous experimental evidence for electron transfer reaction rate maxima at large donor-acceptor separations for several reasons. For example, the donor-acceptor distance must be kept constant on the timescale of the electron transfer events if the influence of reactant separation distance on $\mathrm{k}_{\mathrm{ET}}$ is to be explored. To eliminate diffusion, frozen (glassy) matrices or covalent donor-bridge-acceptor molecules are viable possibilities, but in both cases the choice of suitable donor, bridge, and acceptor components is not straightforward when aiming to search for this effect. Moreover, the solvent plays a key role because the outer-sphere reorganization energy is of pivotal importance. In covalently linked systems, care must be taken regarding increasing $\pi$-conjugation with increasing bridge length. Prior studies of oligo- $p$-phenylene and $p$-phenylene vinylene bridged systems have reported an increase of $\mathrm{k}_{\mathrm{ET}}$ with increasing donor-acceptor distance in some selected cases, ${ }^{[19,20]}$ but this is most likely due to the increasing $\pi$-conjugation of these particular bridges when they are lengthened. ${ }^{[3]}$ The decrease in HOMO-LUMO energy gap leads to smaller barriers for tunneling (stronger superexchange coupling), and in some extreme cases may even result in a changeover from the tunneling to the hopping mechanism. Thus, even when an increase of $\mathrm{k}_{\mathrm{ET}}$ with increasing distance is observed, it is not a priori clear that the observed effect can indeed be attributed to the interplay between $\lambda_{\mathrm{o}}$ and $\mathrm{H}_{\mathrm{DA}}$ illustrated in Fig. $4 b$.

Factors that favor the observation of the effect described herein are the following: (i) a high driving-force (very negative $\left.\Delta \mathrm{G}_{\mathrm{ET}}{ }^{0}\right)$, (ii) relatively low distance decay constant (small $\beta$ ). High driving-forces are readily accessible for thermal chargerecombination reactions which follow photoinduced charge-separation in covalent donor-bridge-acceptor molecules. As molecular bridges, $p$-xylene spacers could be well suited because the $\pi$-conjugation increases much less with increasing bridge length than in $p$-phenylenes and $p$-phenylene vinylenes, ${ }^{[3,19]}$ yet $p$-xylenes provide relatively low $\beta$-values. ${ }^{[21]}$

It has been noted that the two-sphere model (Eqn. (6)) as well as more sophisticated models significantly underestimate the increase of the outer-sphere reorganization energy with increasing distance, at least in some cases. ${ }^{[16]}$ If the increase in $\lambda$. is indeed substantially stronger than anticipated based on theory, this would be beneficial for observation of electron transfer rate maxima at large donor-acceptor separations. In this context, consideration of proton-coupled electron transfer (PCET) reactions might be useful because PCET can be associated with particularly large reorganization energies. ${ }^{[22]}$ One possibility would be to use electron acceptors that can be protonated (or strongly hydrogen-bonded) by the solvent upon electron transfer, or electron donors which are deprotonated in the course of oxidation.

Note added in proof: After submission of this manuscript, we published two experimental studies in which we observed electron transfer rate maxima at large donor-acceptor distances. ${ }^{[23,24]}$

\section{Acknowledgements}

Support from the Swiss National Science Foundation (grant number 200021_146231/1) for research on fundamental aspects of photoinduced electron transfer is gratefully acknowledged.

Received: July 15, 2015

[1] H. B. Gray, J. R. Winkler, Ann. Rev. Biochem. 1996, 65, 537 .

[2] M. Cordes, B. Giese, Chem. Soc. Rev. 2009, 38, 892.

[3] W. B. Davis, W. A. Svec, M. A. Ratner, M. R. Wasielewski, Nature 1998, 396, 60.

[4] H. B. Gray, J. R. Winkler, Proc. Natl. Acad. Sci. U. S. A. 2005, 102, 3534.

[5] B. S. Brunschwig, S. Ehrenson, N. Sutin, J. Am. Chem. Soc. 1984, 106, 6858.

[6] M. Tachiya, S. Murata, J. Phys. Chem. 1992, 96, 8441.

[7] R. A. Marcus, N. Sutin, Biochim. Biophys. Acta 1985, 811, 265.

[8] R. A. Marcus, J. Chem. Phys. 1956, 24, 966

[9] G. L. Closs, J. R. Miller, Science 1988, 240, 440.

[10] L. S. Fox, M. Kozik, J. R. Winkler, H. B. Gray, Science 1990, 247, 1069.

[11] M. R. Wasielewski, M. P. Niemczyk, W. A. Svec, E. B. Pewitt, J. Am. Chem. Soc. 1985, 107, 1080

[12] N. Sutin, Acc. Chem. Res. 1982, 15, 275.

[13] H. M. McConnell, J. Chem. Phys. 1961, 35, 508.

[14] B. Giese, Acc. Chem. Res. 2000, 33, 631.

[15] S. Murata, M. Tachiya, J. Phys. Chem. 1996, $100,4064$.

[16] S. S. Isied, A. Vassilian, J. F. Wishart, C. Creutz, H. A. Schwarz, N. Sutin, J. Am. Chem. Soc. 1988, 110, 635 .

[17] J. G. Kirkwood, F. H. Westheimer, J. Chem. Phys. 1938, 6, 506

[18] E. H. Yonemoto, G. B. Saupe, R. H. Schmehl, S. M. Hubig, R. L. Riley, B. L. Iverson, T. E. Mallouk, J. Am. Chem. Soc. 1994, 116, 4786.

[19] E. A. Weiss, M. J. Ahrens, L. E. Sinks, A. V. Gusev, M. A. Ratner, M. R. Wasielewski, J. Am. Chem. Soc. 2004, 126, 5577.

[20] C. Stangel, C. Schubert, S. Kuhri, G. Rotas, J. T. Margraf, E. Regulska, T. Clark, T. Torres, N. Tagmatarchis, A. G. Coutsolelos, D. M. Guldi, Nanoscale 2015, 7, 2597.

[21] D. Hanss, M. E. Walther, O. S. Wenger, Coord. Chem. Rev. 2010, 254, 2584.

[22] M. Sjödin, S. Styring, B. Åkermark, L. C. Sun, L. Hammarström, J. Am. Chem. Soc. 2000, 122, 3932.

[23] M. Kuss-Petermann, O. S. Wenger, Angew. Chem. Int. Ed. 2016, 55, 815.

[24] M. Kuss-Petermann, O. S. Wenger, J. Am. Chem. Soc. 2016, 138, 1349. 